\title{
Prevalence of Dementia and Associated Factors among Older Adults in Latin America during the COVID-19 Pandemic
}

\author{
Marcio Soto-Añari ${ }^{\mathrm{a}}$ Loida Camargo $^{\mathrm{b}, \mathrm{c}}$ Miguel Ramos-Henderson ${ }^{\mathrm{d}}$ \\ Claudia Rivera-Fernández ${ }^{e}$ Lucia Denegri-Solís ${ }^{e}$ Ursula Calle $^{f}$ Nicanor Mori ${ }^{f}$ \\ Ninoska Ocampo-Barbág Fernanda López ${ }^{\text {h }}$ Maria Porto $^{\mathrm{i}}$ Nicole Caldichoury-Obando $^{\mathrm{j}}$ \\ Carol Saldías $^{k}$ Pascual Gargiulol Cesar Castellanos ${ }^{m}$ Salomon Shelach-Bellido ${ }^{a}$ \\ Norman López ${ }^{i}$
}

\begin{abstract}
aLaboratorio de Neurociencia, Departamento de Psicología, Universidad Católica San Pablo, Arequipa, Peru;

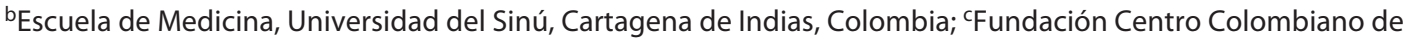
Epilepsia y Enfermedades Neurológicas Jaime Fandiño Franky (FIRE), Cartagena de Indias, Colombia; ${ }^{d}$ Centro de Investigación e Innovación en Gerontología Aplicada (CIGAP), Facultad de Salud, Universidad Santo Tomás,

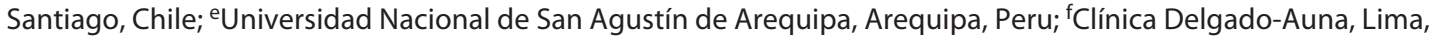
Peru; IInstituto de Neurociencias Comportamentales (INCC), Universidad Autónoma Gabriel René Moreno (UAGRM), Santa Cruz de la Sierra, Bolivia; ${ }^{\text {h}}$ Hospital Nacional Dr. Alejandro Posadas, Buenos Aires, Argentina; ${ }^{\prime}$ Universidad de La Costa, Barranquilla, Colombia; 'Departamento de Ciencias Sociales, Universidad de Los Lagos, Osorno, Chile; ${ }^{k}$ Facultad de Ciencias de la Salud, Universidad San Sebastián, Valdivia, Chile; 'Laboratorio de Neurociencias y Psicología Experimental, CONICET, Facultad de Ciencias Médicas, Universidad Nacional de Cuyo, Mendoza,

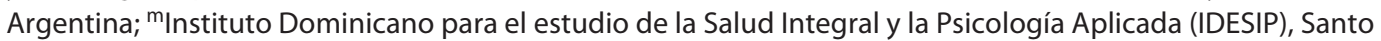
Domingo, Dominican Republic
\end{abstract}

\section{Keywords}

Dementia · Prevalence · Aging · Ethnicity · COVID-19

\begin{abstract}
Background: The COVID-19 pandemic has had a great impact on cognitive health in Latin American older adults, increasing the risk of cognitive impairment and dementia. Our objective was to analyze the prevalence of dementia and the associated factors in Latin American older adults during SARS-CoV-2 pandemic. Methods: A multicentric first phase cross-sectional observational study was conducted during the SARS-CoV-2 pandemic. Five thousand two hundred and forty-five Latin American adults over 60 years of age were studied in 10 countries: Argentina, Bolivia, Chile, Colombia,
\end{abstract}

Ecuador, Guatemala, Mexico, Peru, the Dominican Republic, and Venezuela. We used the telephone version of Montreal Cognitive Assessment, the "Alzheimer Disease 8" scale for functional and cognitive changes, and the abbreviated version of the Yesavage depression scale. We also asked for sociodemographic and lockdown data. All the evaluation was made by telephone. Cross-tabulations and $x^{2}$ tests were used to determine the variability of the prevalence of impairment by sociodemographic characteristics and binary logistic regression to assess the association between dementia and sociodemographic factors. Results: We observed that the prevalence of dementia in Latin America is 15.6\%, varying depending on the country (Argentine $=7.83$ and Bolivia $=28.5 \%$ ). The variables most associated with dementia were race and age. It does not seem to be associated with the pan-
C 2021 The Author(s).

Published by S. Karger AG, Basel

This is an Open Access article licensed under the Creative Commons Attribution-NonCommercial-4.0 International License (CC BY-NC) (http://www.karger.com/Services/OpenAccessLicense), applicable to the online version of the article only. Usage and distribution for commercial purposes requires written permission.
Correspondence to:

Marcio Soto-Añari, msoto@ucsp.edu.pe 
demic but with social and socio-health factors. Conclusion: The prevalence of dementia shows a significant increase in Latin America, attributable to a constellation of ethnic, demographic, and socioeconomic factors.

(C) 2021 The Author(s).

Published by S. Karger AG, Basel

\section{Introduction}

The coronavirus today has caused $>2$ million deaths in the world [1]. Some Latin American countries are among those that report the highest number of infections and deaths from the pandemic (Brazil, Argentina, and Peru). The poor implementation of intermediate and intensive care units, the lack of mechanical ventilators and oxygen, and an inefficient vaccine program; dramatically increased the number of deaths in many Latin American countries [2].

This health crisis has severely impacted all age-groups but with much greater force in the elderly [3]. During the restriction measures due to the pandemic, medical and hospital care services were suspended, as well as complementary nonpharmacological, physical, social, and cognitive intervention services. In some countries, there have been implemented telemedicine services, which have helped many older adults in the clinical follow-up [4]. Unfortunately, these services have not been implemented in all countries or have not had homogeneous coverage [5]. Despite this, the first reports showed higher indicators of mood alterations $[6,7]$, complaints of cognitive decline [8], and increased consultations for neuropsychiatric problems $[9,10]$.

As we can see, restrictions in their daily activities, together with the indicators of more precarious health [11] and the impact on the well-being because the pandemic, could increase significantly the risk of developing dementia [12]. Previous studies in Latin America have shown ranges of dementia between $1.8 \%$ and $11.5 \%[13,14]$. Recent reviews show that the global prevalence of dementia for the region is $11 \%$, which is higher than that registered in other parts of the world [15]. This prevalence is influenced by an important combination of ethnic, risk factors (e. g., low educational level and age), and socioeconomic factors [16]. At the moment, we do not know the prevalence of dementia during the COVID-19 pandemic in Latin America.

As it may be observed, the risk of dementia could increase significantly in Latin America during the pandemic, associated not only with well-known risk factors but also with emotional and sociodemographic factors. To date, no studies have been reported showing the prevalence of dementia in the context of a pandemic and the associated sociodemographic factors. Therefore, we have proposed to analyze the prevalence of dementia and the main associated factors in older Latin American adults during the SARS-CoV-2 pandemic.

\section{Materials and Methods}

\section{Study Design and Sampling}

The study reports the findings of a first phase cross-sectional observational study (screening), carried out during the SARSCoV-2 pandemic in 5,245 Latin American older adults over 60 years of age in 10 countries: Argentina, Bolivia, Chile, Colombia, Ecuador, Guatemala, Mexico, Peru, the Dominican Republic, and Venezuela. The sampling was nonprobabilistic and was obtained from private and public clinics and associations of older adults. The exclusion criteria were not been testing positive for SARSCoV-2 infection, prior clinical diagnosis of dementia or mild cognitive impairment, and without clinical diagnosis of depression or anxiety during the lockdown period (shown in Fig. 1).

Procedure

A group of Latin American researchers and clinicians was formed in April 2020. Between April and May, the measures and protocols to be used were designed, prepared, and tested. The protocol included cognitive, functional, and emotional evaluations and was piloted in May 2020 with 680 participants. Minor changes in linguistic and cultural adaptations were made (e.g., "pieza" [room] in Chile). Simultaneously, study directors together with the country coordinators trained the interviewers in the formats and surveys of the protocol, in addition to the use of the platform to complete the data. All the processes were made online, and every interviewer sends their first full protocol and received a personal feedback from country coordinator. All participants completed about $40 \mathrm{~min}$ of individual evaluation via telephone. Additionally, we evaluated a family member with functional and cognitive tests. The evaluations were carried between July and October 2020. We used the National Institute of Aging criteria for dementia [17] and were carried out in 2 phases. A first one was of screening, considering the cutoff points of the telephone version of Montreal Cognitive Assessment (T-MoCA) [18], and "Alzheimer Disease 8" (AD8) [19]. A second part consisted of the review and validation of the presumptive diagnosis by neuropsychologists and neurologists.

\section{Instruments}

Participants completed sociodemographic, clinical, lifestyle, mood, cognitive, and functional questionnaires, in addition to information associated with the quarantine. For the evaluation of the emotional state, we used the abbreviated version of the Yesavage geriatric depression scale [20], which has shown acceptable indicators of validity for the detection of mood alterations [21]. We used a cutoff level of 2 points to considered indicators of emotional disturbance.

For the cognitive assessment, we elected the T-MoCa, which has shown important indicators of sensitivity and specificity for 
Fig. 1. Flow diagram showing patient in-

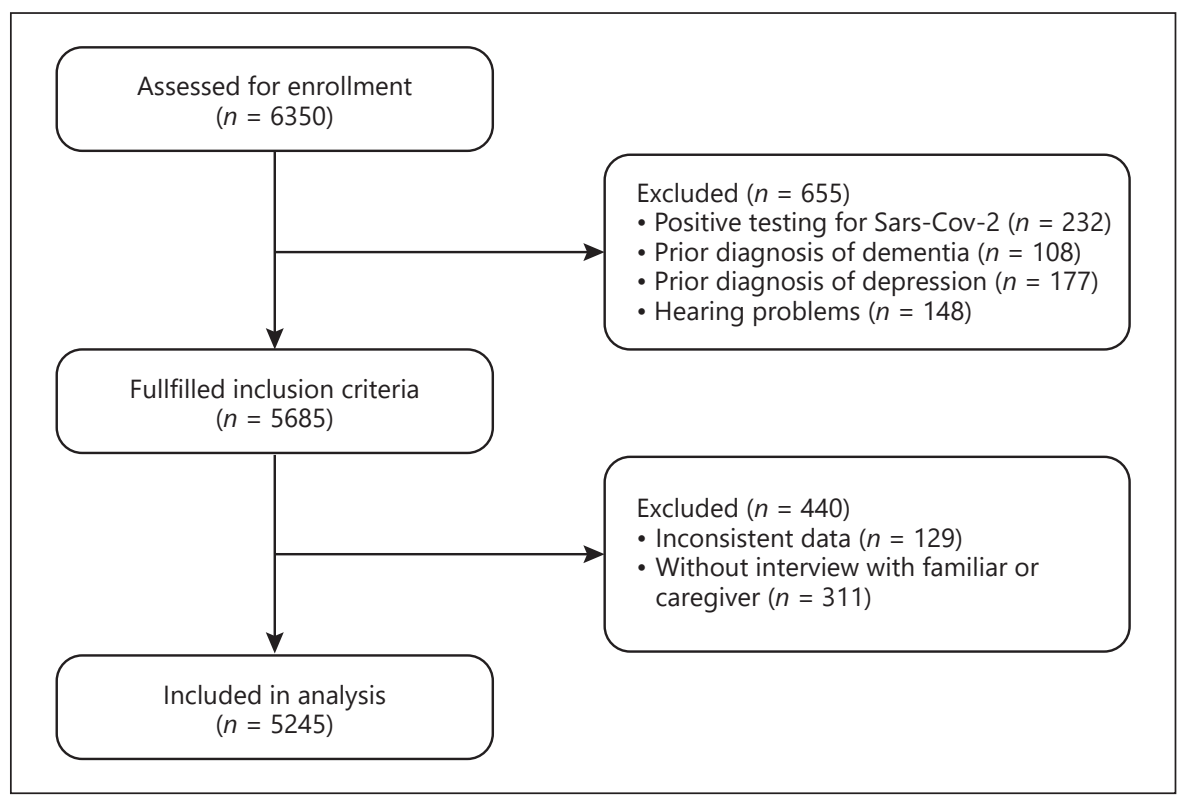
volvement.

cognitive alterations [18] and an important concurrent validity with the full version [22]. In Latin America, the MoCa test is the more frequently brief cognitive test used in clinical settings [23]. It contains 5 items that assess memory, fluency, and orientation. The cutoff level of 11 points has been considered.

Additionally, we used the AD8 to evaluate changes in cognitive and functional domains usually altered in dementia syndrome [24]. It has shown a high degree of sensitivity to cognitive impairment when applied to caregiver [19] and adequate when self-completed [25]. The cutoff point of 2 points has been considered [19]. Finally, we used a questionnaire about the quarantine that included items of level of accomplish, days in quarantine, and beginning day of lockdown, which varies according to the country and the propagation of the SARS-CoV-2 virus in the region.

\section{Statistical Analysis}

Descriptive statistics were used to indicate the most representative sociodemographic characteristics as well as the scores obtained in the cognitive and functional measurements. We obtained the data of deterioration prevalence considering the cutoff points of the T-MoCa and AD8. Once the prevalence percentages were obtained, cross-tabulations and $\chi^{2}$ tests were performed to determine the variability of the prevalence of deterioration due to sociodemographic characteristics. Finally, binary logistic regression was applied to evaluate the association between cognitive impairment and sociodemographic factors, adjusted for age, years of schooling, and the Yesavage scale score. The analyses were performed with the SPSS 25 statistical software synchronized with JASP. The significance level used was $p<0.05$.

\section{Ethical Considerations}

All procedures contributing to this work complied with the ethical standards of the relevant National and Institutional Committees on human experimentation and considering the Declaration of Helsinki. All participants or familiar/caregiver were informed of the study and gave their consent online. The research was approved by the Ethics Committee of the coordinate institution of the project, The Universidad de la Costa (Act no.: 080, research project code: INV.140-02-003-15).

\section{Results}

\section{Sociodemographic and Quarantine Characteristics of the Participants}

The final sample included 5,245 participants. The age range of the sample was between 60 and 94 years old, with a mean of $69.61(\mathrm{SD}=7.28)$. Almost a third of the total sample was women (67\%), with the exception of Peru, which exhibits more homogeneous indicators. The highest proportion of race was Latin American mestizo (55.1\%), which varies depending on the country (Argentina, 1.2\%; Peru, $86.9 \%)$, and white (39.4\%) with ranges between $8.9 \%$ for Peru and $98.8 \%$ for Argentina. More than half of the participants are married (52.3\%). Likewise, the level of education achieved was mostly high school (38.7\%), followed by technical formation (25\%). Regarding the pandemic, almost all of the subjects reported having been in quarantine $(86.1 \%)$ and $>70 \%$ reported having complied duly with the official confinement measures (see Table 1).

\section{Prevalence of Dementia Globally and by Country}

Table 2 shows that the prevalence of dementia for the total sample is $15.6 \%$. We observe that Bolivia and Peru have the highest frequencies of deterioration $(28.5 \%$ and 


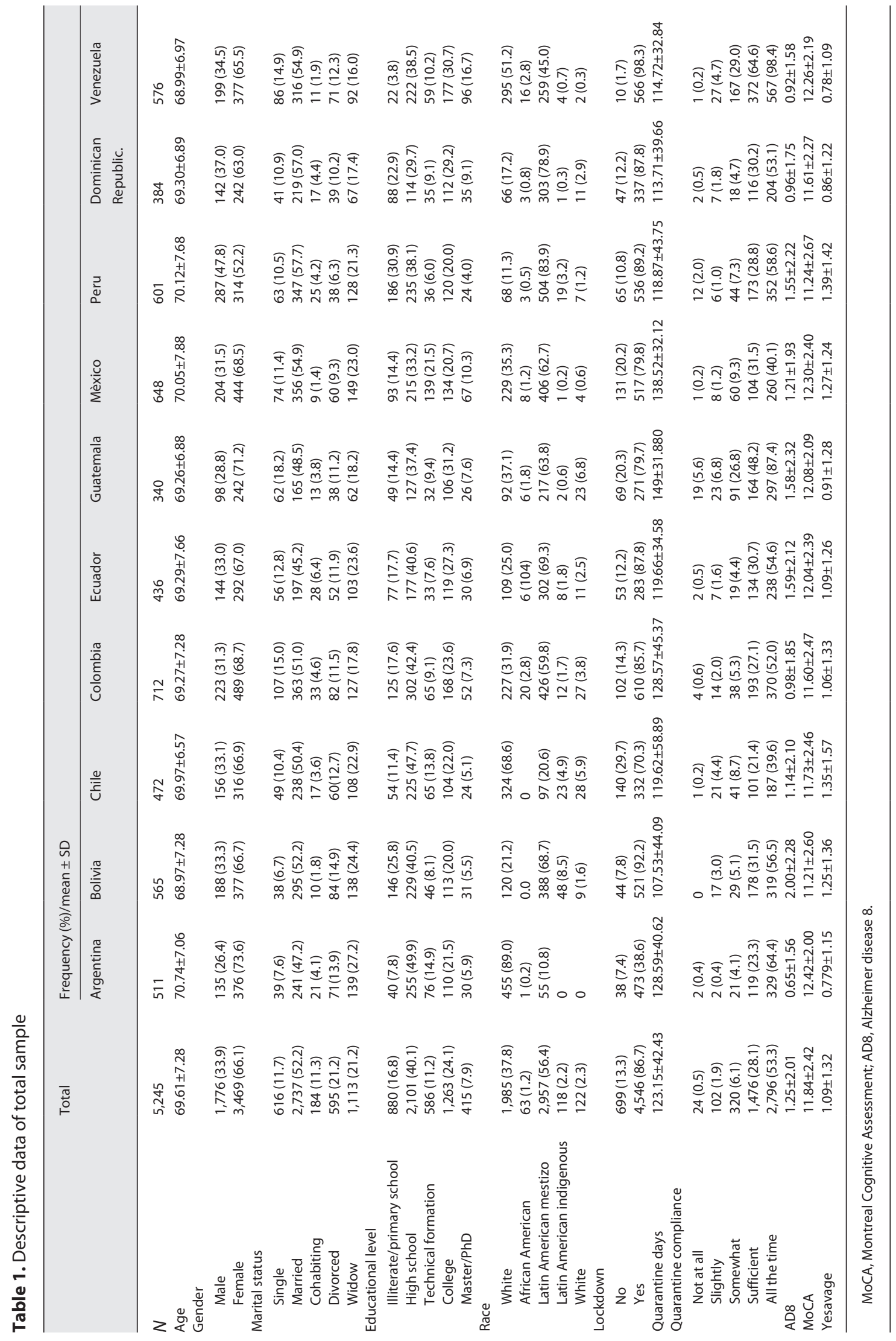


Table 2. Prevalence of dementia by country and total

\begin{tabular}{lll}
\hline Country & $\begin{array}{l}\text { Without dementia, } \\
n(\%)\end{array}$ & $\begin{array}{l}\text { With dementia, } \\
n(\%)\end{array}$ \\
\hline Argentina & $471(92.17)$ & $40(7.83)$ \\
Bolivia & $404(71.50)$ & $161(28.50)$ \\
Chile & $396(83.90)$ & $76(16.10)$ \\
Colombia & $620(87.08)$ & $92(12.92)$ \\
Ecuador & $364(83.49)$ & $72(16.51)$ \\
Guatemala & $282(82.94)$ & $58(17.06)$ \\
Mexico & $581(89.66)$ & $67(10.34)$ \\
Peru & $457(76.04)$ & $144(23.96)$ \\
Dominican Republic & $339(88.28)$ & $45(11.72)$ \\
Venezuela & $513(89.06)$ & $63(10.94)$ \\
\hline Total sample & $4,427(84.40)$ & $818(15.60)$ \\
\hline
\end{tabular}

24\%, respectively), while Argentina and Mexico show the lowest (7.83\% and $10.34 \%$, respectively).

Prevalence of Dementia by Age, Sex, and Race

We observe that cognitive impairment varies significantly by age and race. Specifically, we see that dementia is higher in older participants, reaching $52.8 \%$ among adults between 90 and 94 years old, while in the 60-64 age-groups, it is $9.6 \%$. Likewise, we see that the prevalence of dementia is higher among Latin American Indian people $(34.7 \%)$ than the other racial groups (see Table 3).

\section{Risk Factors for Dementia}

Multivariate analysis reveals that the risk of dementia (odds ratio) in African Americans is 2.2 times higher and in Latin American Indians it is 1.8 times higher than in the other participants. Likewise, we see that at an older age the probability increases significantly. Subjects between 65 and 69 years are 1.3 times more likely to have dementia, while those between 90 and 94 years are 9.5 times more likely. Similarly, we found that the emotional state increases the risk by 1.2 times. The analysis by country shows that there is a higher probability of CI in participants from Bolivia (4 times higher risk), Guatemala (2.9 times higher risk), Peru (2.6 times higher risk), and Ecuador (2.1 times higher risk) to a lesser extent, Chile (1.6) and Venezuela (1.7). Finally, we observed that quarantine did not increase the risk of cognitive impairment in the sample studied (see Table 4).

\section{Discussion}

Our purpose was to analyze the prevalence of dementia in Latin America during the SARS-CoV-2-associated pandemic. Our results show a global prevalence of $15.6 \%$ of presumptive cases of dementia in older adults without COVID-19 infection, varying according to the country (higher prevalence in Bolivia and Peru and lower in Argentina and Mexico). In addition, we observed an important relationship with age, level of education, ethnicity, and emotional state.

The prevalence found is higher than that reported in other studies $[13,15]$. Specifically, Zurique found prevalence with ranges between $2 \%$ and $42 \%$, which varied according to the type of study, age of the sample, and diagnostic criteria. Likewise, Nitrini et al. [13] reported a standardized prevalence of 7.1 (ranges between 2.66 and 12.16) but that it increased significantly among illiterates (total prevalence: 15.67, ranges between 5.03 and 36.06). In both cases, a significant variability was observed between countries.

Our data also show important difference between countries. The highest prevalence is found in those countries where social and health systems are the weakest in the region (Bolivia, Peru, and Guatemala) and have indicators of higher health risk such as being overweight or even basic sanitation [26] but also in those that show a higher proportion of the aging population (Chile) [27]. As we can see, Latin America is one of the regions with the highest indicators of inequality in access to health care. Inhabitants with low socioeconomic status, from rural areas and of different ethnic groups, have very limited access to care and indicators of higher health risk [26]. These factors, together with those associated with the pandemic (isolation, greater health neglect), are leading to a major health crisis among older adults, which as we see is exacerbating emotional and cognitive alterations [28].

On the other hand, the data show that the risk of dementia increases significantly with advancing age. We observe that the risk can be 9 times higher among adults aged 90-95 years than between 60 and 64 years. Epidemiological studies have consistently described this pattern [29]. On the other hand, years of schooling have been shown to be a protective factor in different studies [30]. This could be associated with the cognitive reserve effect generated by having higher levels of schooling [31]. However, in Latin American samples, years of schooling seem not to be a good predictor of cognitive performance [32] due to the enormous variability in educational quality in the region. 
Table 3. Prevalence of dementia by age, sex, and race

\begin{tabular}{llll}
\hline & Without dementia, $n(\%)$ & With dementia, $n(\%)$ & $X^{2}$ \\
\hline $\begin{array}{l}\text { Age-group } \\
60-64\end{array}$ & $1,384(90.39)$ & $147(9.60)$ & $258.270^{* *}$ \\
$65-69$ & $1,241(87.58)$ & $176(12.42)$ & $151(14.45)$ \\
$70-74$ & $894(85.55)$ & $144(21.62)$ & \\
$75-79$ & $522(78.38)$ & $99(27.50)$ & 0.928 \\
$80-84$ & $261(72.50)$ & $82(43.16)$ & \\
$85-89$ & $108(56.84)$ & $19(52.78)$ & \\
$90-94$ & $17(47.22)$ & $265(14.92)$ & \\
Gender & $1,511(85.08)$ & $553(15.94)$ & \\
Male & $2,916(84.06)$ & $265(13.35)$ & \\
Female & $1,720(86.65)$ & $11(17.46)$ \\
Race & $52(82.54)$ & $484(16.37)$ & \\
White & $2,473(83.63)$ & $41(34.45)$ \\
African American & $77(65.25)$ & $17(13.93)$ & \\
Latin American mestizo & $105(86.07)$ & \\
Latin American indigenous & & \\
Another & & \\
\hline ** $p<0.001$. & & \\
\hline
\end{tabular}

Likewise, we observed that ethnicity has a significant relationship with a greater probability of deterioration, especially in African Americans and Latin American Indians. These results have been seen in studies with ethnic minorities $[33,34]$ and also in population studies in Latin America [35]. These results could be associated with factors linked to the social determinants of health, which combine a series of factors that include a low educational level, greater vascular problems (more cases of diabetes and hypertension), poor eating habits, among others, and that have shown an important effect on health [16]. Finally, we see that the emotional state has a significant impact on the risk of cognitive impairment. As we have seen previously, there is an important effect of the pandemic overemotional state [6] and an altered cognition, as founded in the longitudinal study [36]. It has been considered as a preclinical phase of dementia [37].

It is interesting to note that the factors associated with the pandemic (days of quarantine and the level of accomplish) did not seem to affect the cognitive state of the sample studied. This could be associated with the level of compliance with the government's measures, which in many countries was not total. The need to carry out activities to generate economic income, due to the high degree of informality of the economy [38] and given that the majority of Latin American older adults live with their family [39], led to maintaining some levels of activity, providing them emotional support during quarantine.

Despite the interesting results found, this study is not exempt of limitations. In first place, considering that it is a cross-sectional study based on the cutoff points of the screening tests and clinical judgment for diagnosis, it is possible to fall in misdiagnosis. However, we have taken the gold standard criteria for dementia diagnosis [17] and follow the guidelines of the handbook for best practices in dementia diagnosis in the Latin America region [40]. Therefore, it is essential to monitor the sample and corroborate the diagnosis. Second, it is important to validate the instruments used. The MoCa test is one of the most widely used instruments in Latin America [23, 41] but its use has been extended to the pencil and paper version. The short version and application via telephone or telemedicine ( $\mathrm{T}-\mathrm{MoCa}$ ) have not yet been validated in the region. Despite this, studies in other contexts [42] have shown significant convergent validity of the full version. On the other hand, in the case of $\mathrm{AD} 8$, we used the criteria of the Cochrane review [43] and the Chilean version as a reference [19]. Therefore, its validation is necessary in the other countries of the region.

These psychometric aspects of the instruments are fundamental, especially in the current pandemic circumstances where telemedicine and teleneuropsychology have become important for the monitoring and 
Table 4. Associated factors to dementia

\begin{tabular}{|c|c|c|c|c|c|}
\hline \multirow[b]{2}{*}{ Ages of schooling } & \multirow{2}{*}{$\begin{array}{l}\begin{array}{l}\text { Standard } \\
\text { error }\end{array} \\
0.008\end{array}$} & \multirow{2}{*}{$\begin{array}{c}\begin{array}{c}\text { Odds } \\
\text { ratio }\end{array} \\
0.907\end{array}$} & \multirow{2}{*}{$\begin{array}{l}p \text { value } \\
<0.001\end{array}$} & \multicolumn{2}{|c|}{$\begin{array}{l}95 \% \text { confidence interval } \\
\text { (odds ratio scale) }\end{array}$} \\
\hline & & & & 0.892 & 0.923 \\
\hline Yesavage & 0.031 & 1.248 & $<0.001$ & 1.176 & 1.328 \\
\hline \multicolumn{6}{|l|}{ Race } \\
\hline \multicolumn{6}{|l|}{ Whites (RC) } \\
\hline African American & 0.375 & 2.143 & 0.028 & 1.074 & 4.708 \\
\hline Latin American mestizo & 0.107 & 0.868 & 0.184 & 0.704 & 1.070 \\
\hline Latin American indigenous & 0.242 & 1.818 & 0.013 & 1.131 & 2.920 \\
\hline Another & 0.315 & 0.757 & 0.377 & 0.408 & 1.405 \\
\hline \multicolumn{6}{|l|}{ Age-group } \\
\hline \multicolumn{6}{|l|}{ 60-64 (RC) } \\
\hline $65-69$ & 0.131 & 1.324 & 0.033 & 1.023 & 1.713 \\
\hline $70-74$ & 0.138 & 1.396 & 0.017 & 1.061 & 1.824 \\
\hline $75-79$ & 0.143 & 2.161 & $<0.001$ & 1.629 & 2.868 \\
\hline $80-84$ & 0.164 & 3.140 & $<0.001$ & 2.271 & 4.342 \\
\hline $85-89$ & 0.191 & 4.944 & $<0.001$ & 3.385 & 7.220 \\
\hline $90-94$ & 0.378 & 9.591 & $<0.001$ & 4.753 & 20.113 \\
\hline \multicolumn{6}{|l|}{ Quarantine compliance } \\
\hline \multicolumn{6}{|l|}{ Not at all (RC) } \\
\hline Slightly & 0.734 & 2.087 & 0.316 & 0.495 & 8.802 \\
\hline Somewhat & 0.685 & 2.785 & 0.135 & 0.727 & 10.674 \\
\hline Sufficient & 0.669 & 2.834 & 0.119 & 0.764 & 10.509 \\
\hline All the time & 0.666 & 3.027 & 0.096 & 0.821 & 11.157 \\
\hline Gender (female) & 0.095 & 0.840 & 0.067 & 0.701 & 1.016 \\
\hline \multicolumn{6}{|l|}{ Country } \\
\hline \multicolumn{6}{|l|}{ Argentina (RC) } \\
\hline Bolivia & 0.216 & 4.092 & $<0.001$ & 2.677 & 6.254 \\
\hline Chile & 0.246 & 1.644 & 0.044 & 1.012 & 2.671 \\
\hline Colombia & 0.224 & 1.470 & 0.087 & 0.986 & 2.376 \\
\hline Ecuador & 0.237 & 2.158 & 0.001 & 1.354 & 3.441 \\
\hline Guatemala & 0.244 & 2.961 & $<0.001$ & 1.834 & 4.780 \\
\hline Mexico & 0.238 & 1.181 & 0.483 & 0.742 & 1.882 \\
\hline Peru & 0.220 & 2.618 & $<0.001$ & 1.696 & 4.043 \\
\hline Dominican Republic & 0.256 & 1.638 & 0.081 & 0.991 & 2.707 \\
\hline Venezuela & 0.228 & 1.719 & 0.018 & 1.099 & 2.688 \\
\hline
\end{tabular}

$\mathrm{RC}$, reference category.

evaluation of older adults. They could also help generate protocols to serve the rural population and ethnic minorities that do not have access to specialists in cognitive impairment [44]. On the other hand, it is necessary to increase the representativeness of ethnic minorities and rural areas. Our results show a higher prevalence of dementia in ethnic minorities but the sample size is low and the presence of many confounding factors is high. Finally, it would be convenient to analyze the positive cases for COVID and assess the consequences in the medium- and long-term. Recent reviews show neurological alterations [45], so it is important to monitor this population group.

Dementia in Latin America during COVID-19

\section{Conclusions}

We can conclude that the prevalence of cognitive impairment shows a significant increase in Latin America, which varies depending on the country. The variables most associated with this increased risk are race, gender, and age but it seems not to be associated with the pandemic and if it is associated with social and socio-health factors. This first multicenter study in the context of a pandemic shows the need to follow-up older adults and provide contexts for evaluation, monitoring, and intervention to avoid a significant increase in cases. Furthermore, it highlights the importance of conducting multi-

Dement Geriatr Cogn Disord Extra 2021;11:213-221 219 
national studies that allow us to standardize criteria for evaluation, diagnosis, and follow-up.

\section{Statement of Ethics}

All procedures followed the ethical standards on human experimentation according to the Declaration of Helsinki. All participants or familiar/caregiver were informed of the study and gave their consent orally. The research was approved by the Ethics Committee of the coordinate institution of the project, the Universidad de la Costa (Act no.: 080, research project code: INV.140-02003-15).

\section{Conflict of Interest Statement}

The authors have no conflicts of interest to declare.

\section{Funding Sources}

This study did not receive any funding.

\section{Author Contributions}

Marcio Soto-Añari and Norman López made substantial contribution to the conception, design, analysis, and interpretation of data, revised the manuscript for important intellectual content, and approved the final version. Loida Camargo contributed to analysis and interpreted data, revised the manuscript for intellectual content, and approved the final manuscript. Miguel RamosHenderson contributed to the design, methods, analysis, and interpretation of data, revised the entire manuscript, and approved the final manuscript. Claudia Rivera-Fernandez and Ninoska Ocampo-Barba contributed to acquisition, analysis, and interpretation of data, revised the manuscript for important intellectual content, and approved the final version. Lucia Denegri-Solís, Ursula Calle, Nicanor Mori, Fernanda López, María Porto, Nicole Caldichoury-Obando, Carol Saldias, and Cesar Castellanos contributed to acquisition, analysis, and interpretation of data, revised the manuscript, and approved the final version. Pascual Gargiulo contributed to analysis and interpretation of data, revised the manuscript for important intellectual content, and approved the final version. Salomon Shelach-Bellido contributed to analysis and interpretation of data, revised the manuscript, and approved the final version.

\section{Data Availability Statement}

The data that support the findings of this study can be acquired from the corresponding author upon reasonable request.

\section{References}

1 World Health Organization. Coronavirus disease (COVID-19) dashboard [Internet]. 2021 [cited 2021 Apr 13]. Available from: https://covid19.who.int/table.

2 Pan American Health Organization. Time is of the essence - countries of the Americas must act now to slow the spread of COVID-19 [Internet]. 2020 [cited 2021 Apr 13]. Available from: https://www.paho.org/hq/ index.php?option =com_content \& view=article \& id=15762: time-is-of-the-essence-countries-of-the-americas-must-actnow-to-slow-the-spread-of-covid-19\& Itemid=1926\&lang=en.

3 Ibanez A, Kosik KS. COVID-19 in older people with cognitive impairment in Latin America. Lancet Neurol. 2020;19(9):719-21.

4 Murphy RP, Dennehy KA, Costello MM, Murphy EP, Judge CS, O’Donnell MJ, et al. Virtual geriatric clinics and the COVID-19 catalyst: a rapid review. Age Ageing. 2020; 49(6):907-14.

5 Gray DM, Joseph JJ, Olayiwola JN. Strategies for digital care of vulnerable patients in a COVID-19 world - keeping in touch. JAMA Health Forum. 2020;1(6):e200734.
6 Soto-Añari M, Ramos-Henderson MA, Camargo L, Calizaya López J, Caldichoury N, López N. The impact of SARS-CoV-2 on emotional state among older adults in Latin America. Int Psychogeriatr. 2021;33(2):1934.

7 Moreno C, Wykes T, Galderisi S, Nordentoft $\mathrm{M}$, Crossley N, Jones N, et al. How mental health care should change as a consequence of the COVID-19 pandemic. Lancet Psychiatry. 2020;7(9):813-24.

8 van Maurik IS, Bakker ED, van den Buuse S, Gillissen F, van de Beek M, Lemstra E, et al. Psychosocial effects of corona measures on patients with dementia, mild cognitive impairment and subjective cognitive decline. Front Psychiatry. 2020;11:585686.

9 Manca R, De Marco M, Venneri A. The impact of COVID-19 infection and enforced prolonged social isolation on neuropsychiatric symptoms in older adults with and without dementia: a review. Front Psychiatry. 2020; 11:585540.
10 Sorbara M, Graviotto HG, Lage-Ruiz GM, Turizo-Rodriguez CM, Sotelo-López LA, Serra A, et al. COVID-19 and the forgotten pandemic: follow-up of neurocognitive disorders during lockdown in Argentina. COVID-19 y la pandemia olvidada: el seguimiento de las enfermedades neurocognitivas durante la cuarentena en Argentina. Neurologia. 2021; 36(1):9-15.

11 Rockwood K, Howlett SE. Fifteen years of progress in understanding frailty and health in aging. BMC Med. 2018;16(1):220.

12 Livingston G, Huntley J, Sommerlad A, Ames D, Ballard C, Banerjee S, et al. Dementia prevention, intervention, and care: 2020 report of the Lancet Commission. Lancet. 2020; 396(10248):413-46.

13 Nitrini R, Bottino CM, Albala C, Custodio Capuñay NS, Ketzoian C, Llibre Rodriguez JJ, et al. Prevalence of dementia in Latin America: a collaborative study of population-based cohorts. Int Psychogeriatr. 2009;21(4):62230.

14 Molero AE, Pino-Ramírez G, Maestre GE. High prevalence of dementia in a Caribbean population. Neuroepidemiology. 2007;29(12):107-12. 
15 Zurique Sánchez C, Cadena Sanabria MO, Zurique Sánchez M, Camacho López PA, Sánchez Sanabria M, Hernandez Hernandez $\mathrm{S}$, et al. [Prevalence of dementia in the elderly in Latin America: a systematic review]. Rev Esp Geriatr Gerontol. 2019;54(6):346-55. Spanish.

16 Baez S, Ibáñez A. Dementia in Latin America: an emergent silent Tsunami. Front Aging Neurosci. 2016;8:253.

17 McKhann GM, Knopman DS, Chertkow H, Hyman BT, Jack CR, Kawas CH, et al. The diagnosis of dementia due to Alzheimer's disease: recommendations from the National Institute on Aging-Alzheimer's Association workgroups on diagnostic guidelines for Alzheimer's disease. Alzheimers Dement. 2011; $7(3): 263-9$.

18 Horton DK, Hynan LS, Lacritz LH, Rossetti HC, Weiner MF, Cullum CM. An abbreviated montreal cognitive assessment (MoCA) for dementia screening. Clin Neuropsychol. 2015;29(4):413-25.

19 Muñoz C, Núñez J, Flores P, Behrens PMI, Slachevsky A. [Usefulness of a brief informant interview to detect dementia, translated into Spanish (AD8-Ch)]. Rev Med Chil. 2010; 138(8):1063-5. Spanish.

20 Gómez-Angulo C, Campo-Arias A. Escala de yesavage para depresión geriátrica (GDS-15 y GDS-5): estudio de la consistencia interna y estructura factorial. Univ Psychol. 2011; 10(3):735-43.

21 Pocklington C, Gilbody S, Manea L, McMillan D. The diagnostic accuracy of brief versions of the geriatric depression scale: a systematic review and meta-analysis. Int J Geriatr Psychiatry. 2016;31(8):837-57.

22 Liew TM. The optimal short version of montreal cognitive assessment in diagnosing mild cognitive impairment and dementia. J Am Med Dir Assoc. 2019;20(8):1055.e1-e8.

23 Custodio N, Duque L, Montesinos R, AlvaDiaz C, Mellado M, Slachevsky A. Systematic review of the diagnostic validity of brief cognitive screenings for early dementia detection in Spanish-speaking adults in Latin America. Front Aging Neurosci. 2020;12:270.
24 Chen HH, Sun FJ, Yeh TL, Liu HE, Huang HL, Kuo BI, et al. The diagnostic accuracy of the Ascertain Dementia 8 questionnaire for detecting cognitive impairment in primary care in the community, clinics and hospitals: a systematic review and meta-analysis. Fam Pract. 2018;35(3):239-46.

25 Chin R, Ng A, Narasimhalu K, Kandiah N. Utility of the AD8 as a self-rating tool for $\operatorname{cog}$ nitive impairment in an Asian population. Am J Alzheimers Dis Other Demen. 2013; 28(3):284-8.

26 OECD/The World Bank. Panorama de la salud: latinoamérica y el caribe. Paris: OECD Publishing; 2020 [cited 2021 Apr 12].

27 Albala C. El envejecimiento de la población chilena y los desafíos para la salud y El bienestar de las personas mayores. Rev Méd Clín Las Condes. 2020;31(1):7-12.

28 Vahia IV, Jeste DV, Reynolds CF 3rd. Older adults and the mental health effects of COVID-19. JAMA. 2020;324(22):2253-4.

29 Sibbett RA, Russ TC, Deary IJ, Starr JM. Risk factors for dementia in the ninth decade of life and beyond: a study of the Lothian birth cohort 1921. BMC Psychiatry. 2017;17(1): 205.

30 Stern Y. Cognitive reserve in ageing and Alzheimer's disease. Lancet Neurol. 2012;11(11): 1006-12.

31 Arce Rentería M, Vonk JMJ, Felix G, Avila JF, Zahodne LB, Dalchand E, et al. Illiteracy, dementia risk, and cognitive trajectories among older adults with low education. Neurology. 2019;93(24):e2247-56.

32 Soto-Añari M, Flores-Valdivia G, FernándezGuinea S. [Level of reading skills as a measure of cognitive reserve in elderly adults]. Rev Neurol. 2013;56(2):79-85. Spanish.

33 Lipnicki DM, Makkar SR, Crawford JD, Thalamuthu A, Kochan NA, Lima-Costa MF, et al. Determinants of cognitive performance and decline in 20 diverse ethno-regional groups: a COSMIC collaboration cohort study. PLoS Med. 2019;16(7):e1002853.

34 Vega IE, Cabrera LY, Wygant CM, Velez-Ortiz D, Counts SE. Alzheimer's disease in the latino community: intersection of genetics and social determinants of health. J Alzheimers Dis. 2017;58(4):979-92.
35 Mayeda ER, Glymour MM, Quesenberry CP Whitmer RA. Inequalities in dementia incidence between six racial and ethnic groups over 14 years. Alzheimers Dement. 2016; 12(3):216-24.

36 Makizako H, Shimada H, Doi T, Tsutsumimoto K, Hotta R, Nakakubo S, et al. Comorbid mild cognitive impairment and depressive symptoms predict future dementia in community older adults: a 24-month followup longitudinal study. J Alzheimers Dis. 2016; 54(4):1473-82.

37 Steffens DC. Late-life depression and the prodromes of dementia. JAMA psychiatry. 2017; 74(7):673-4.

38 Benítez MA, Velasco C, Sequeira AR, Henríquez J, Menezes FM, Paolucci F. Responses to COVID-19 in five Latin American countries. Health Policy Technol. 2020;9(4):52559.

39 Palloni A, Pinto G. Family support networks and population ageing in latin america and the caribbean. Int J Sociol Fam. 2014;40(2): 199-203.

40 Ibáñez A, Slachevsky A, Serrano C. Manual de buenas prácticas para el diagnóstico de demencias. BID; 2020.

41 Loureiro C, Garcia C, Adana L, Yacelga T, Rodriguez-Lorenzana A, Maruta C. [Use of the montreal cognitive assessment (MoCA) in Latin America: a systematic review]. Rev Neurol. 2018;66(12):397-408. Spanish.

42 Carlew AR, Fatima H, Livingstone JR, Reese C, Lacritz L, Pendergrass C, et al. Cognitive assessment via telephone: a scoping review of instruments. Arch Clin Neuropsychol. 2020; 35(8):1215-33.

43 Hendry K, Green C, McShane R, Noel-Storr AH, Stott DJ, Anwer S, et al. AD-8 for detection of dementia across a variety of healthcare settings. Cochrane Database Syst Rev. 2019; 3(3):CD011121.

44 Parra MA, Baez S, Allegri R, Nitrini R, Lopera F, Slachevsky A, et al. Dementia in Latin America: assessing the present and envisioning the future. Neurology. 2018;90(5):222-31.

45 Whittaker A, Anson M, Harky A. Neurological manifestations of COVID-19: a systematic review and current update. Acta Neurol Scand. 2020;142(1):14-22. 